\title{
ENHANCING THE STRUCTURAL STABILITY OF LQ OPTIMAL CONTROLLERS VIA THE MCS ADAPTIVE ALGORITHM
}

\author{
Mario di Bernardo* Stefania Santini ${ }^{* *, 1}$ \\ ${ }^{*}$ Dipartimento di Ingegneria \\ Università del Sannio in Benevento \\ Piazza Roma 18 - 82100 Benevento, Italy. \\ ** Dipartimento di Informatica e Sistemistica \\ Università degli Studi di Napoli Federico II \\ via Claudio, 21 - 80125 Napoli, Italy.
}

\begin{abstract}
This paper discusses the implementation of an LQ control strategy through the use of a Minimal Control Synthesis adaptive algorithm recently presented in the literature. It is shown that by using such approach the structural stability of the closed-loop system can be improved with respect both parameter mismatches and nonlinear perturbations. A case study discussed in the literature on LQ controllers is used to illustrate the strategy presented in the paper. Copyright (C)2005 IFAC
\end{abstract}

Keywords: Optimal Control; Minimal Control Synthesis; Adaptive Control; Structural Stability.

\section{INTRODUCTION}

Often, in applications it is required for the controller to minimize certain cost criteria while control is attained. It is now common practice in applications to address this requirement by using classical Optimal Control techniques such as the well-known Linear Quadratic Regulators (LQR) (Anderson and Moore, 1971). It has been shown that, typically, LQ schemes lack the flexibility and the structural stability of other more sophisticated control approaches as, for instance, exemplified by the two significative cases discussed in (Soroka and Shaked, 1984) and (Zang and Fu, 1996). The lack of robustness to model uncertainties and nonlinear perturbation is, at times, a strong limitation for the use of LQ strategies. In facts, many problems of relevance in applications contain uncertainties, model inaccuracies and other effects that can make the use of classical optimal control schemes unviable.

\footnotetext{
1 Corresponding author: stsantin@unina.it
}

One way of achieving greater control flexibility is to use adaptive control schemes where the control gains can be appropriately varied according to the system behavior. Recently, a novel model-reference adaptive algorithm has been presented which relies on minimal knowledge of the plant (Stoten and Benchoubane, 1990a). The scheme, which has been termed as Minimal Control Synthesis Algorithm (or MCS), has been shown to be robust to nonlinear terms and slowly varying parameter variations (Stoten and Benchoubane, $1990 b)$.

From an application viewpoint, it would be desirable to merge the optimal approach with an adaptive scheme in order to guarantee optimality of the control action while maintaining some of the benefits of an adaptive strategy. Most of the schemes attempting the integration of adaptive and optimal control approaches presented in the literature sofar require intense computations, good knowledge of the plant and the absence of any nonlinear perturbation on the plant model (see for example (Rusnak and Guez, 1995), (El-Farra and 


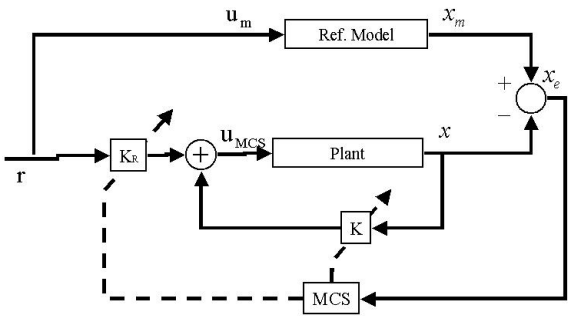

Figure 1. The MCS adaptive control scheme

Christofides, 1999)). Thus, despite their potential benefits, such controllers are hardly used in applications as they are too costly to design and implement.

The aim of this paper is to propose a simple implementation of an optimal LQ controller based on the use of a minimal control synthesis adaptive algorithm. We will show that in so doing it is possible to implement the LQ strategy on the plant even in those cases where the LQ strategy by itself has been shown to lack structural stability. The choice of the MCS controller allows the control objective to be achieved with minimal knowledge of the plant and with little design effort as the adaptive gains are started from zero initial conditions.

We will present two alternative MCS based optimaladaptive schemes. The effectiveness of the proposed strategies is validated on the example discussed in (Soroka and Shaked, 1984) to illustrate the lack of robustness of classical LQ controllers. We will show that the LQ-MCS implementation introduced in this paper is indeed effective in maintaining stability and achieve acceptable sub-optimal solutions even when a solely LQ action would fail. Moreover a proof of the asymptotic stability of the resulting closed-loop plant is provided in section 3.1 .

\section{MCS ALGORITHM: A BRIEF OVERVIEW}

The MCS algorithm was first introduced in (Stoten and Benchoubane, 1990a) as an extension of the Landau Model Reference Adaptive scheme. The MCS strategy relies on minimal knowledge of the plant dynamics. Namely, it is assumed that the controlled system (plant) is controllable and observable and it has unknown parameters but a known phase canonical structure

$$
\dot{x}(t)=A x(t)+B u(t),
$$

The main aim of MCS control is for the plant states, $x(t)$, to track asymptotically the states, $x_{m}(t)$, of a given reference model of the form (Stoten and Benchoubane, 1990a)

$$
\dot{x}_{m}(t)=A_{m} x_{m}(t)+B_{m} r(t)
$$

with $r(t)$ being some desired reference signal.

As shown in Fig. 1, the MCS control input, say $u_{M C S}(t)$, consists of a feed-forward and a feedback action with time-varying adaptive gains defined as

$$
u_{M C S}(t)=K(t) x(t)+K_{R}(t) r(t)
$$

with

$$
\begin{aligned}
K(t) & =\alpha \int_{0}^{t} y_{e}(\tau) x^{T}(\tau) d \tau+\beta y_{e}(t) x^{T}(t) \\
K(0) & =K_{0}, K \in \mathbb{R}^{n} \\
K_{R}(t) & =\alpha \int_{0}^{t} y_{e}(\tau) r(\tau) d \tau+\beta y_{e}(t) r(t) \\
K_{R}(0) & =K_{R 0}, K_{R} \in \mathbb{R}
\end{aligned}
$$

and $\alpha$ and $\beta$ being positive scalar adaptation weights; $y_{e}$ is the output error computed as

$$
y_{e}(t)=C_{e} x_{e}(t),
$$

where

$$
\begin{aligned}
& x_{e}(t)=x_{m}(t)-x(t), \\
& C_{e}=\left[\begin{array}{llll}
0 & \ldots & 0 & 1
\end{array}\right] P,
\end{aligned}
$$

and $\mathrm{P}$ is the solution of the Lyapunov equation

$$
P A_{m}+A_{m}^{T} P=-M, \quad M>0 .
$$

Note that typically the adaptive gains are started from zero, i.e. $K_{0}=0$ and $K_{R 0}=0$.

As shown in (Stoten and Benchoubane, 1990a), the MCS controller can be proven to guarantee asymptotic stability of the error system, with the plant states tracking asymptotically the states of the reference model. Moreover, the MCS algorithm was also shown to be robust again rapidly varying disturbances and (unmodelled) nonlinear perturbations of the form $f(x)=$ $\left[\begin{array}{lll}0 \cdots 0 & d(x, t)\end{array}\right]^{T}$ with $d(x, t)$ acting on the plant dynamics (Stoten and di Bernardo, 1996).

\section{AN LQ-MCS IMPLEMENTATION}

In order, to address the robustness properties of classical control schemes while maintaining the simplicity of use of the MCS algorithm and its benefit, we shall seek now to integrate a classical LQ optimal control approach with the MCS algorithm.

The main idea is to use the MCS to concretely provide to the real plant the control input generated by a classical LQ optimal controller acting on a nominal model of the plant. In so doing any mismatch between the nominal model and the real plant will be compensated by the adaptive action of the MCS, which will also guarantee stability in those cases where the LQ strategy alone would fail.

We propose to implement the MCS scheme on the real plant selecting as a reference model, the nominal model of the plant controlled via a classical LQ optimal strategy, i.e. choosing $u_{m}(t)$ in figure 1 as an optimal control input.

The steps required to build up this scheme can be summarized as follows. 


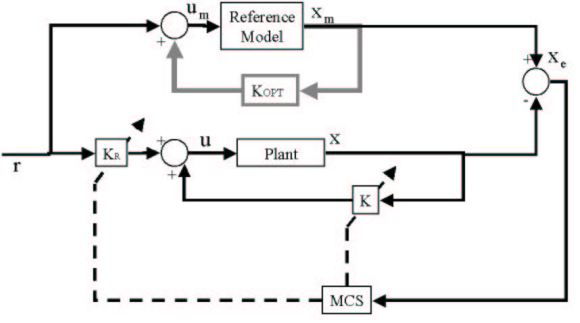

Figure 2. Optimal Reference Minimal Control Synthesis (scheme (a)).

(1) Identify a nominal linear model of the plant of interest of the form:

$$
\dot{x}_{0}(t)=A_{0} x_{0}(t)+B_{0} u_{0}(t)
$$

Note that thanks to the robustness properties of the MCS, we do not need to take explicitly into account nonlinear terms acting on the plant or parameter uncertainties. The model above represents a rough estimate of the plant matrices that can be used to synthesize a classical optimal control law.

(2) Synthesize a classical LQ optimal controller on the nominal plant model. Note that the optimal controlled nominal plant (8) can be also rewritten as

$$
\dot{x}_{0}(t)=\left(A_{0}+B_{0} K^{o p t}\right) x_{0}(t)+B_{0} r(t),
$$

with optimal control gains $K^{o p t}$ or equivalently

$$
\dot{x}_{0}(t)=A_{m} x_{0}(t)+B_{m} r(t) .
$$

(3) Implement the LQ-MCS scheme by using the closed-loop LQ nominal plant as the reference model for the MCS adaptive controller acting on the real plant given by:

$$
\begin{aligned}
\dot{x}(t)= & \left(A_{0}+\Delta A\right) x(t)+\left(B_{0}+\Delta B\right) u(t)= \\
& =A x(t)+B u(t)
\end{aligned}
$$

where the matrices $\Delta A, \Delta B$ model parameter uncertainties on the real plant dynamics.

Figure 2 describes the LQ-MCS scheme.

\subsection{Proof of asymptotic stability}

In order to put our new generalization in context, a proof of asymptotic stability of the LQ-MCS algorithm is given in this section. The main idea behind the proof is that of writing the model following error dynamics as a feedback system with a strictly positive real (SPR) forward path and a nonlinear feedback path which satisfies the Popov Criterion as given by (14).

We first write the error equation from (10), (11), (3) as

$$
\begin{aligned}
\dot{x}_{e}=\left(\dot{x}_{0}-\dot{x}\right)= & A_{m} x_{e}(t)+\left[A_{m}-A-B K(t)\right] x(t)+ \\
& +\left[B_{m}-B K_{R}(t)\right] r(t)
\end{aligned}
$$

then, after some algebraic manipulation (given the canonical structure of $A$ and $A_{m}$ ), we get

$$
\dot{x}_{e}=A_{m} x_{e}+B_{e} \Phi(t) w(t)
$$

where $\Phi(t)=\left[\Phi_{x} \Phi_{R}\right] ; \Phi_{x}(t)=\left[\Phi_{1} \cdots \Phi_{n}\right]=$ $\left[A_{m}-A-B K(t)\right]_{n} ; \Phi_{R}(t)=\left[B_{m}-B K_{R}(t)\right]_{n} ; w(t)=$ $\left[\begin{array}{llll}x_{1} & \cdots & x_{n}(t) & r(t)\end{array}\right]^{T}$.

Therefore the error system represented in (13) can be rewritten as a nonlinear feedback system. We now wish to demonstrate that this closed loop system will be globally asymptotically stable and that the error $x_{e}(t)$ will tend to zero with time. This proof is achieved in two steps which may be described heuristically as follows:

(1) Show that the forward path, the linear system formed by the triple $A_{m}, B_{e}, C_{e}$ is passive, i.e. a system that never creates energy. This condition is also referred to as the system transfer function $H(s)=C_{e}^{-1}\left(s I-A_{m}\right) B_{e}$ being strictly positive real (SPR). For a given transfer function to be SPR, it must satisfy the following conditions: (a) $H(s)$ must be strictly stable; (b) the real part of $H(s)$ must be strictly positive along the imaginary axis, i.e. $\forall \omega \geq 0, \quad \operatorname{Re}\{h(j \omega)\}>0$.

(2) Prove that the feedback block will only ever produce a finite amount of energy. This can be shown using the Popov's Criterion.

So, if these two conditions are satisfied then any energy produced by the adaptive feedback block will be eventually dissipated by the forward path and as a consequence the global error output will tend to zero as time goes to infinity.

The first condition can be verified by the KalmanYakubovitch Lemma, as reported in (Stoten and Benchoubane, $1990 a$ ).

For the second condition to be satisfied, the feedback block must verify the integral inequality

$$
I=\int_{t_{1}}^{t_{2}} y_{e}(t)[-\Phi(t) w(t)] d t \geq-c^{2}
$$

for all $t_{2} \geq t_{1}$, where $c \in \mathbb{R}$ is a constant independent of $t_{2}$.

From equations (4), we can write each gain term as the sum of an integral (cf. $\alpha$ ) and a proportional contribution (cf. $\beta$ ):

$$
K_{i}(t)=K_{i \alpha}(t)=K_{i \alpha}(t)+K_{i \beta}(t), \quad i=1,2, \cdots, N
$$

and similarly

$$
\Phi_{i}(t)=\Phi_{i \alpha}(t)=\Phi_{i \alpha}(t)+\Phi_{i \beta}(t), \quad i=1,2, \cdots, N
$$

Notice that if we assume that the variation of the system parameters is significantly slower than the adaptation evolution, we can deduce that:

$$
\dot{\Phi}_{i \alpha}(t) \approx-b \alpha y_{e}(t) w_{i}(t)=\frac{\alpha}{\beta} \Phi_{i \beta}
$$


By decomposing the integral term $I$ in (14) as

$$
\int_{t_{1}}^{t_{2}} y_{e}(t)[-\Phi(t) w(t)] d t=\sum_{i} I_{i \alpha}+\sum I_{i \beta}
$$

then (14) is satisfied if each of the component integrals $I_{i \alpha}=\int_{t_{1}}^{t_{2}} y_{e}(t)\left[-\Phi_{i \alpha}(t) w_{i}(t)\right] d t$ and

$I_{i \beta}=\int_{t_{1}}^{t_{2}} y_{e}(t)\left[-\Phi_{i \beta}(t) w_{i}(t)\right] d t$ satisfies the Popov inequality.

By adopting an equality in (15) we can then write

$$
\begin{array}{r}
I_{i \alpha}=\frac{1}{b \alpha} \int_{t_{1}}^{t_{2}}\left[\Phi_{i \alpha}(t) \dot{\Phi}_{i \alpha}\right] d t= \\
=\frac{1}{2 b \alpha}\left[\Phi_{i \alpha}^{2}(t)\right]_{t_{1}}^{t_{2}} \geq \frac{1}{2 b \alpha} \Phi_{i \alpha}^{2}\left(t_{1}\right)=-c_{i \alpha}^{2} .
\end{array}
$$

with $c_{i \alpha} \in \mathbb{R}$.

Moreover

$$
I_{i \beta}=\int_{t_{1}}^{t_{2}} \Phi_{i \beta}^{2}(t) d t \geq 0
$$

Hence the second condition is proven.

This, together with the fact that the triple $A_{m}, B_{e}, C_{e}$ is SPR guarantees the asymptotic stability of the closedloop plant.

We remark that the stability analysis of the closedloop system has been investigated in absence of nonlinear perturbations such as unmodelled nonlinear dynamics. Robustness with respect to unknown nonlinear dynamics acting on the real plant (11) as

$$
\dot{x}(t)=A x(t)+B u(t)+f(x)
$$

is guaranteed by the MCS algorithm (see (Stoten and Benchoubane, 1990b) for the proof).

\section{AN ALTERNATIVE IMPLEMENTATION OF THE LQ-MCS APPROACH}

In this section we suggest a possible alternative implementations of our LQ-MCS strategy (see figure 3). Mainly the difference between this schemes and the one proposed in section 3 is in the computation of the control input to the plant.

In particular, in the first scheme (scheme (a), figure 2) the control input to the real plant is entirely provided by the MCS controller, i.e. it is selected as in (3). In this second scheme (scheme (b), figure 3), instead, this is complemented with a further contribution from the optimal LQ input to the nominal model of the plant, i.e. $u_{M C S}(t)+u_{O P T}(t)$. While in the former case, the MCS provides the entire control effort, in the second scheme the role of the MCS action is to adjust the optimal LQ control low by means of adaptive perturbations.

In this paper the effectiveness of this second control implementation is investigated through simulations. Results and a numerical comparison between the two schemes can be found in section 5 .

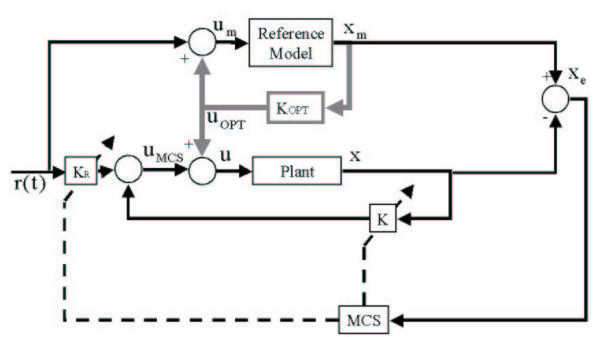

Figure 3. Minimal Control Synthesis with Optimal Feed-Forward action (scheme (b)).

\section{A REPRESENTATIVE EXAMPLE}

As mentioned above classical LQ controllers are typically effective only if the plant parameters are perfectly known in the absence of nonlinear perturbations. It has been shown that the presence of parameter mismatches in the plant can even cause a complete loss of stability under LQ control. A particularly striking case was discussed in (Soroka and Shaked, 1984) (see (Zang and Fu, 1996) and references therein for other examples). In those cases where stability is still preserved, often poor control performances are observed in terms of higher values of the cost function (see for example (Xue et al., 1999)).

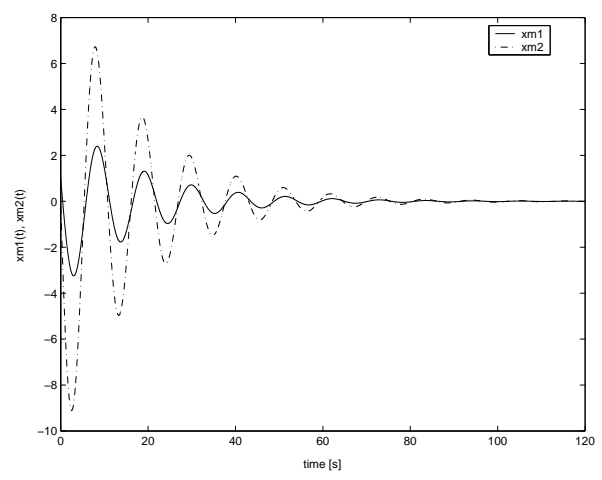

Figure 4. Closed-loop LQ nominal plant: time history of the state variables. Solid line: $x_{m 1}(t)$. Dash-dot line: $x_{m 2}(t)$.

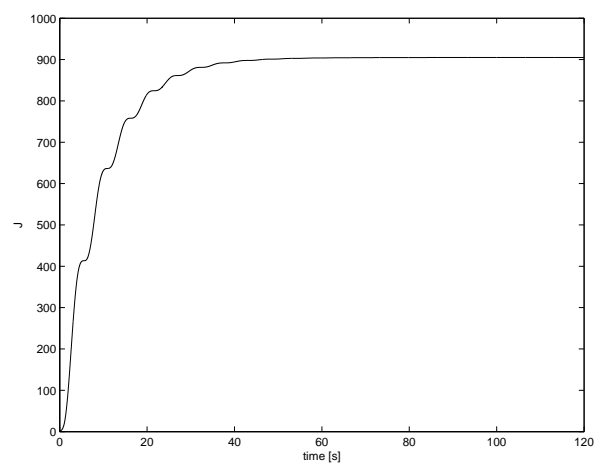

Figure 5. Closed-loop LQ nominal plant: time history of the cost function.

To validate the proposed LQ-MCS approach, we will discuss in this section its application to the representative case described in (Soroka and Shaked, 1984). As mentioned in (Soroka and Shaked, 1984), despite of its 


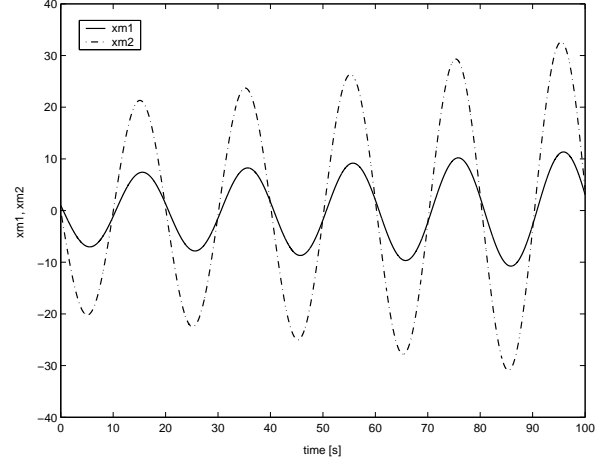

Figure 6. Closed-loop LQ real plant in presence of a mismatch in the model parameters: time history of the state variables. Solid line: $x_{m}(1)(t)$. Dashdot line: $x_{m}(2)(t)$.

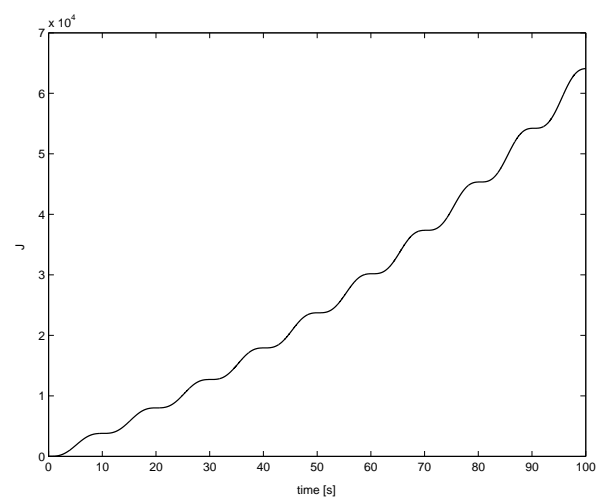

Figure 7. Closed-loop LQ real plant in presence of a mismatch in the model parameters: time history of the cost function.

impressive margins, full state linear quadratic optimal regulator may suffer from robustness problems where small changes in the parameters of the system may lead to fast unstable closed-loop behaviour. Note that, as remarked in (Soroka and Shaked, 1984), the lack of robustness is also observed for small parameter variations in the normalized input matrix and does not depend on the diagonality of the control weighting matrix.

Namely, we consider the synthesis of an LQ state feedback optimal regulator for the single input single output linear time-invariant nominal model of the plant of the form (10), where

$$
A_{m}=\left(\begin{array}{cc}
-1 & 0 \\
0 & -2
\end{array}\right), \quad B_{m}=\left(\begin{array}{l}
1 \\
2
\end{array}\right), \quad C_{m}=\left(\begin{array}{ll}
1 & 1
\end{array}\right)
$$

We assume that the controller should be found that minimizes the performance index, $J$, defined as

$$
J=\int_{0}^{\infty}\left[y_{m}^{2}(t)+r u^{2}(t)\right] d t, \quad r>0
$$

As shown in (Soroka and Shaked, 1984), the optimal control gains can be found to be given by $K=\left(\begin{array}{ll}k_{1} & k_{2}\end{array}\right)$, where $k_{1}=1+q-\sqrt{5+2 q}$ and $k_{2}=$ $2 \sqrt{5+2 q}-q-4$ with $q=\sqrt{4+1 / r}$.
The stability region reported in (Soroka and Shaked, 1984), shows that small variations in the system parameters can cause the optimal controller to induce unstable dynamics on the closed-loop system. We consider for example the case when $r=0.01$. With this choice of $r$, the optimal gains for the nominal plant (8) are $K=(6.16,4.12)$. In Figs. $4-7$, we see that the optimal controller successfully achieve the output regulation to zero, guaranteeing minimality of the performance index (with an asymptotic value $J_{\infty} \approx 900$ as depicted in Fig 5).

Now assume that the same optimal controller is implemented on the real plant which we assume is described by (11) with

$$
\Delta A=\left(\begin{array}{ll}
0 & 0 \\
0 & 0
\end{array}\right), \quad \Delta B=\left(\begin{array}{c}
-0.02 \\
1
\end{array}\right) .
$$

As shown in Fig 6, 7, the LQ control action is now destabilizing, thus, as expected, small parameter variations can cause unexpected losses of structural stability.

We propose to overcome this problem, by making use of the LQ-MCS implementations proposed in this paper (see scheme (b) in figure 3). Figures 8, 9 show the time evolution of the states of the real plant and those of the optimal plant under the effects of the LQMCS strategy. We see that the integration of the LQ controller with an additional MCS adaptive strategy guarantees stability even in the presence of parameter variations. Moreover, the performance index (see Figure 10) stay close to the original value guaranteed by the LQ controller acting on the nominal plant (in Fig. $5)$.

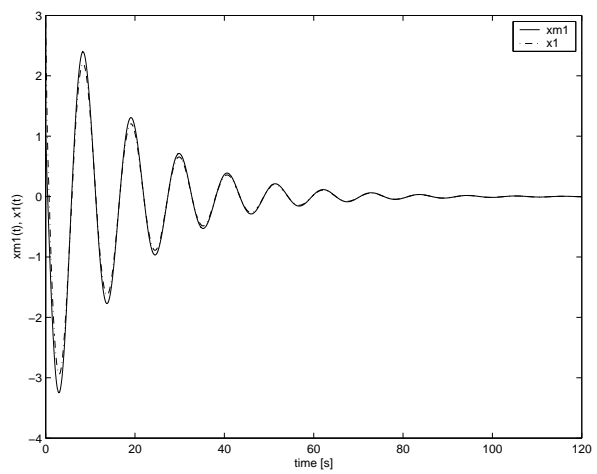

Figure 8. Closed-loop LQ-MCS plant (see scheme (b) in figure 3 ). Time history of the first state variables of the real plant and the reference model, respectively $x_{1}(t), x_{m 1}(t)$.

Similar performances can be achieved by using the control scheme (a) described in Figure 2. The control input in this case is rapidly varying since it is completely provided by the adaptive action This obviously reflects in the state space behavior as can be seen in Figures 11 and 12. 


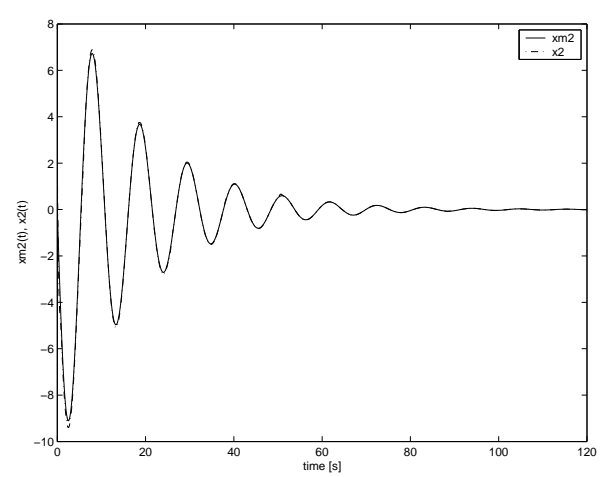

Figure 9. Closed-loop LQ-MCS plant (see scheme (b) in figure 3): time history of the second state variables of the real plant and the reference model, respectively $x_{2}(t), x_{m 2}(t)$.

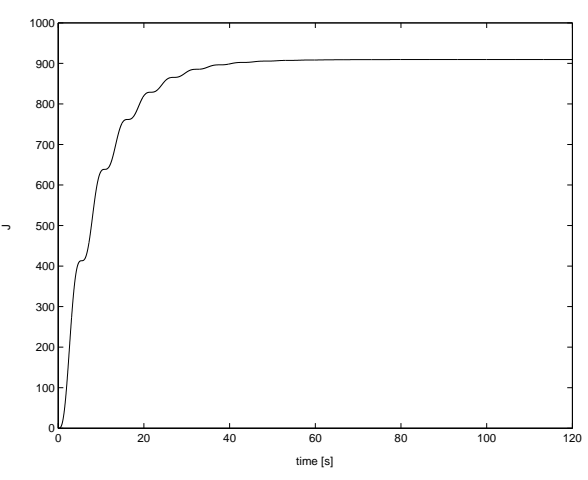

Figure 10. Closed-loop LQ-MCS plant (see scheme (b) in figure 3): time history of the cost function.

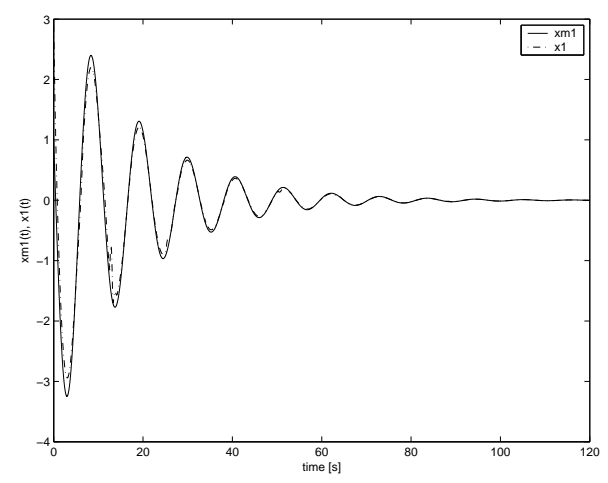

Figure 11. Closed-loop LQ-MCS plant (see scheme (a) in figure 2.): time history of the first state variables of the real plant and the reference model, respectively $x_{1}(t), x_{m 1}(t)$.

\section{CONCLUSIONS}

In this paper we have shown that it is indeed possible to improve the structural stability of classical LQ controllers by integrating them within a novel adaptive control scheme, namely the Minimal Control Synthesis algorithm. Stability of the closed-loop plant has been proofed. By using a representative example presented in the literature to exemplify the lack of robustness of classical LQ controllers, we have also shown the effectiveness of the proposed LQ-MCS implementation. We wish to emphasize that, even if more

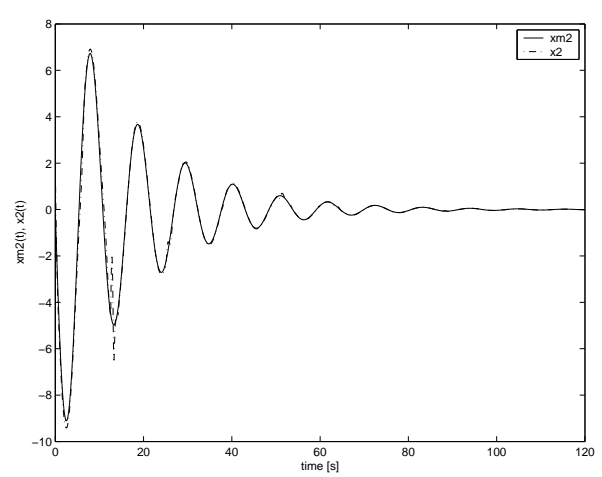

Figure 12. Closed-loop LQ-MCS plant (see scheme

(a) in figure 2.): time history of the second state variables of the real plant and the reference model, respectively $x_{2}(t), x_{m 2}(t)$.

sophisticated solutions can be found, the proposed approach relying on the MCS is simple to design and easy to implement for practical applications.

Ongoing research is focussed towards the formulation of an extended MCS control law including an explicit extra adaptive term to guarantee minimization of an appropriate performance index.

\section{REFERENCES}

Anderson, B. D. O. and J. B. Moore (1971). Linear Optimal Control. Englewood Cliff NJ, Prentice Hall.

El-Farra, N. H. and P. D. Christofides (1999). Robust optimal control of nonlinear systems. Proceedings of the 1999 American Control Conference 1, 124-128.

Rusnak, I. and A. Guez (1995). Optimal adaptive control of uncertain stochastic linear systems. Proc. of American Control Conference 4, 25202524.

Soroka, E. and U. Shaked (1984). On the robustness of LQ regulators. IEEE Transaction on Automatic Control 29(7), 664-665.

Stoten, D. P. and H. Benchoubane (1990a). Empirical studies of an mrac algorithm with minimal controller synthesis. International Journal of Control pp. 823-849.

Stoten, D. P. and H. Benchoubane (1990b). Robustness of a minimal controller synthesis algorithm. International Journal of Control pp. 850-861.

Stoten, D. P. and M. di Bernardo (1996). Application of the minimal control synthesis algorithm to the control and synchronisation of chaotic systems. International Journal of Control pp. 925-938.

Xue, A., Y. Lu and Y. Sun (1999). Robust lq optimal controller designing for refining process. Proceedings of the 1999 IEEE International Conference on Control Applications 1, 403-408.

Zang, C. and M. Fu (1996). A revisit to the gain and phase margins of linear quadratic regulators. IEEE Transaction on Automatic Control 41(10), 1527-1530. 